\title{
MÁS ALLÁ DE LA JUSTICIA CONMUTATIVA: DERECHO DE CONTRATOS, JUSTICIA Y PRECIOS JUSTOS*
}

\author{
Beyond Commutative Justice: Contract Law, \\ Justice, ANd Just Prices
}

JOAQUÍN REYES BARROS**

\begin{abstract}
Resumen:
En este artículo evalúo la teoría del precio justo defendida por James Gordley, según la cual el precio justo es aquel precio que preserva la justicia conmutativa. Luego de oponer algunas objeciones a esta perspectiva, sugiero que enfocarse en la naturaleza institucional de los precios, rechazar el monismo valórico implícito en la versión estándar del precio justo y permitir la posibilidad de un enfoque pluralista a la justificación de los precios puede contribuir a avanzar la teoría del precio justo.
\end{abstract}

Palabras clave: justicia conmutativa; precio justo; igualdad en el intercambio; valor de intercambio; hechos institucionales

\begin{abstract}
:
In this paper I evaluate James Gordley's account of the just price as the price that preserves commutative justice. After raising some objections to this approach, I suggest that focusing on the institutional nature of prices, rejecting the value monism implicit in the standard account of the just price and allowing for the possibility of a pluralist approach to price justification can contribute to moving just price theory forward.
\end{abstract}

Keywords: Commutative Justice; Equality in Exchange; Exchange Value; Institutional Facts

* Agradezco a Amalia Amaya, Chloe Kennedy, Luís Duarte D’Almeida, Alice Krzanich, Ismael Martínez-Torres, Euan MacDonald, Cormac MacAmlaigh, Claudio Michelon, Lucas Miotto-Lopes, Margaret O’Brien, Felipe Oliveira de Sousa, Diego Papayannis, Esteban Pereira Fredes, Alberto Pino Emhart, Alexander Vargas Tinoco, Antonia Walterman, Neil Walker, y a dos críticos anónimos por comentarios y sugerencias útiles a versiones anteriores de este artículo. También quisiera agradecer al público en Lucerne, Girona, Maastricht, Oxford y Edimburgo.

** University of Edinburgh, Reino Unido y Universidad Finis Terrae, Chile (Joaquin. Reyes@ed.ac.uk). Artículo recibido el 14 de marzo de 2020, y aceptado para su publicación el 3 de junio de 2020 . 


\section{INTRODUCCIÓN}

En nuestra experiencia como agentes del mercado, encontramos variadas situaciones en las que el precio de un determinado bien parece 'errado', ya sea porque es exorbitante, 'un robo', 'demasiado', ya, por el contrario, porque es 'una ganga' o 'demasiado bajo'. Sea un profesional extrayendo ganancias enormes por un trabajo que estaría dispuesto a hacer por menos, un vendedor cobrando más por un bien porque sabe cuán intensamente lo quiere el comprador, un comprador aprovechándose de la ignorancia del vendedor para comprar barato un ítem costoso, o incluso un almacén subiendo el precio de sus palas de nieve justo después de una tormenta de nieve, parece ser que el precio pagado por ciertos bienes no se corresponde con el precio que dichos bienes debieran tener, esto es, su precio justo. ${ }^{1}$

Varias reglas legales y prácticas relacionadas con intercambios desiguales y precios injustos en el derecho privado parecen seguir esta intuición. De hecho, la inteligibilidad de reglas e instituciones profundamente arraigadas en el derecho privado dependen de que se establezca algún estándar normativo de precios justos. Los remedios contra la laesio enormis ${ }^{2}$ en el derecho civil continental, la doctrina del trato inicuo [unconscionability] en el common law, ${ }^{3}$ diversos criterios de conducta en la forma de deberes implícitos de trato justo y buena fe, ${ }^{4}$ prohibiciones de especulación de precios, etc., son instancias en que reglas del derecho privado han sido diseñadas teniendo a la vista una preocupación por el hecho de que ciertos bienes están siendo vendidos por más o menos de cuanto debiera ser. $^{5}$

1 El ejemplo de las palas de nieve es tomado de KAHNEMAN et al. (1986), p. 76.

2 Laesio enormis consiste en la compra y venta de bienes por más o menos de una determinada proporción, generalmente la mitad de precio sobre o bajo cierto criterio. Véase en general GoRDLEY (2006), pp. 364 y ss.

3 Sobre la inequidad [unconscionability] del precio, véase en general Deutch (1977), pp. 122-136; GoRDLEY (1991), pp. 154-158; GoRDLEY (2006), p. 365.

4 Véase, por ejemplo, Restatement (Second) of Contracts (1981), §205: “Todo contrato impone sobre cada parte un deber de buena fe y trato justo en su ejecución y en la labor de hacerlo cumplir."

5 Esto no es negar que ha habido signos recientes de una disminución del impacto de la teoría del precio justo en el derecho privado. Véase, por ejemplo, la exclusión explícita de la justa determinación de precios como criterio de justicia en STUDY GrouP \& ACQUis GrouP (2009), p. 225: “II. - 9:406 Examen de exclusiones por injusticia (2): Para los términos de contrato que sean redactados en lenguaje simple e inteligible, la prueba de injusticia no se extiende ni a la definición del tema principal del contrato ni a la adecuación del precio a pagar" (Énfasis añadido). Para su exclusión en los Estados Unidos, véase Restatement (Sec- 
Pero. ¿cuál debiera ser el precio de una cosa? De acuerdo con la versión estándar de la teoría, que puede ser rastreada hasta la doctrina escolástica del iustum pretium, el precio justo es el precio que preserva la justicia conmutativa. Según esta versión, un precio preserva la justicia conmutativa cuando mantiene una igualdad del valor de cambio entre los bienes intercambiados. ${ }^{6}$ Entre los académicos del derecho contemporáneos, esta opinión ha sido defendida y desarrollada por James Gordley, quien afirma que, bajo condiciones ideales de competencia económica, el precio justo correspondería al precio de mercado, porque intercambiar al precio de mercado preservaría la actual distribución de poder adquisitivo.

En este artículo, evalúo y critico esta postura respecto a la justicia en los precios y exploro la posibilidad de una revisión y parcial reformulación de la teoría del precio justo. La perspectiva que propongo se enfoca en la naturaleza institucional de los precios, y sostiene que el precio justo se concibe mejor como el precio que puede ser alcanzado cuando las condiciones de trasfondo del intercambio son justas. Esta concepción del precio justo conlleva un rechazo del monismo valórico implícito en la comprensión tradicional del precio justo y por tanto una aceptación del pluralismo valórico en la justificación de los precios. En el enfoque propuesto, la justicia conmutativa es solo uno entre varios valores que pueden cumplir el propósito de hacer justos los precios.

El artículo se estructura en dos secciones principales. La primera sección explica la versión del precio justo de Gordley y presenta dos objeciones principales a esa versión. La primera objeción consiste en una negación de la afirmación de Gordley de que el precio justo puede preservar la actual distribución del poder adquisitivo. La segunda objeción, y la más importante, cuestiona la fuerza normativa de los precios de mercado, y por tanto, su capacidad para servir como criterio normativo para una determinación justa de los precios.

La segunda sección explora un enfoque alternativo hacia la justicia en la determinación de precios, que tiene como punto de partida la naturaleza institucional de los precios y del sistema de precios. Este enfoque nos permite separar el problema de la justicia en la determinación de precios de preocupaciones sobre justicia conmutativa, al tiempo que abre espacio teórico para un pluralismo de valores en la justificación de los precios, en el que otros valores como la autonomía, la eficiencia o la justicia distributiva pueden tener cabida al responder

ond) of Contracts (1981), §79: "si el requerimiento de consideración se cumple, no hay un requerimiento adicional de equivalencia en el valor intercambiado".

6 Gordley (1981), Gordley (1991), Gordley (2006), Gordley (2001), Gordley (2015), GordLEY y JiANG (2019). 
preguntas sobre justificación de precios. Esta sección no desarrolla una teoría acabada de justificación de precios: solo argumenta a favor de la posibilidad de desarrollar un enfoque de este tipo.

Finalmente, el artículo concluye con algunas observaciones acerca de las implicancias de una perspectiva como la propuesta para nuestra comprensión más general del derecho de contratos.

\section{GORDLEY Y EL PRECIO JUSTO}

Para los teóricos del derecho privado, es casi parte de la descripción de su trabajo el explicar y justificar esquemas legales pre-modernos en relación a preocupaciones modernas sobre autonomía individual o eficiencia económica. ${ }^{7}$ Una rápida revisión de las teorías contemporáneas acerca del derecho privado es suficiente para notar que las teorías deontológicas, basadas en derechos, y las teorías basadas en la eficiencia, son los dos enfoques más comunes para tratar de derecho privado. Esto es también cierto para el derecho de contratos. Las perspectivas deontológicas del derecho de contratos tienden a resaltar la relevancia de que las partes contrayentes respeten los derechos del otro en sus tratos mutuos, mientras que las perspectivas del contrato de tipo Law and Economics típicamente se preocupan más de cómo maximizar la satisfacción de preferencias reduciendo los costos de transacción y encontrando reglas de asignación de riesgos que sean tanto económicamente eficientes como aceptables para ambas partes. ${ }^{8}$

Sin embargo, estas dos perspectivas no cuentan la historia completa acerca del derecho de contratos. James Gordley ha estado defendiendo por casi cuatro décadas que el derecho de contratos moderno debe más a la metafísica aristotélica que a principios kantianos o utilitaristas, y que por tanto es a Aristóteles a quien debemos volver para dar inteligibilidad a nuestras instituciones de derecho privado. En su obra más influyente, The Philosophical Origins of Modern Contract Doctrine, Gordley argumentó largamente que el hecho de que el derecho privado moderno esté organizado a través de las mismas categorías básicas de contrato, daño (tort) y propiedad en todo el mundo, es el resultado del intento consciente

7 Esta tendencia anacrónica en la teoría del derecho privado fue recientemente notada y criticada en GARDNER (2018), p. 197.

8 Para teorías de contrato basadas en la autonomía, véase, por ejemplo, FrIED (2015), Weinrib (1995), Weinrib (2012), Ripstein (2016), Voyiakis (2017), Dagan y Heller (2017). Para teorías de contrato basadas en la eficiencia, véase Kronman y Posner (1979), Posner (2014), Kaplow y ShaVell (2006), Kornhauser (2017). 
de los Escolásticos tardíos de organizar el derecho privado de acuerdo a principios aristotélicos. ${ }^{9}$ Gordley explica que esta articulación de las instituciones del derecho privado se hizo omnipresente en los sistemas legales a través de un proceso de diseminación que empezó con las obras de los iusnaturalistas del norte de Europa del siglo XVII -Grocio, Pufendorf y Barbeyrac-. Aunque estos autores conscientemente rechazaron la filosofía de Aristóteles -o al menos, aquella que tomaron por la filosofía de Aristóteles- ellos, aun así, conservaron la estructura del derecho privado desarrollada por los escolásticos tardíos de acuerdo con dichos principios. Las obras de estos iusnaturalistas influyeron profundamente en juristas franceses del siglo XVIII como Domat y Pothier, y las doctrinas de estos juristas, especialmente las de Pothier, fueron repetidas casi literalmente por el Código Civil Napoleónico. Desde ese momento en adelante pasaron a la mayoría de los sistemas legales alrededor del mundo, incluido el Common Law. En efecto, cuando en el siglo XIX los juristas del derecho anglosajón intentaron comprender el Common Law [Common Law en cursiva] apelando a categorías abstractas, lo hicieron tomando prestado extensamente de las doctrinas continentales de Grocio, Pufendorf, Domat y Pothier.

Los sistemas de derecho privado moldeados sobre principios aristotélicos ahora "gobiernan casi el mundo entero". ${ }^{10}$ Sin embargo, como el mismo Gordley admite, esos principios aristotélicos que ayudaron a dar forma a la doctrina de contratos moderna han caído ahora en desprestigio. El hablar de causas formales, fines últimos, esencia, substancia y accidentes, como también la comprensión de la justicia como una virtud guiada por la sabiduría práctica han sido reemplazados en la teoría del derecho de contratos por un discurso teorético dominado por conceptos como 'maximización de utilidades', 'satisfacción de preferencias', 'autonomía' o 'libertad'. Según Gordley, estos conceptos modernos tienen un poder explicativo muy limitado. Las teorías de derecho de contratos basadas en la autonomía y la eficiencia compartirían el mismo defecto base, a saber, el asumir que los contratos se explican exclusivamente por el hecho de que las partes han elegido algo, sin importar el contenido de aquello que se eligió. Ni la satisfacción de preferencias ni la autonomía son capaces de explicar por qué el derecho a veces no hace cumplir la voluntad de las partes, ni por qué lee en el contrato un conjunto específico de términos -los términos 'naturales' o 'implícitos' del contrato- que las partes nunca aceptaron conscientemente. Cualesquiera sean sus

9 Gordley defiende que la organización de la doctrina de contratos provista por los escolásticos tardíos es tanto (1) el origen de la doctrina de contratos moderna como (2) la mejor explicación de la doctrina de contratos moderna. Véase GordLey (1991), p. 3 y passim. Agradezco a un revisor anónimo por esta observación.

10 Gordley (1991), p. 1. 
méritos, por tanto, estas teorías modernas del contrato no son capaces de explicar algunas características muy básicas del derecho de contratos actualmente existente.

Para resolver este problema, Gordley argumenta que necesitamos volver a las mismas categorías aristotélicas que fueron tan apasionadamente rechazadas en los siglos pasados. ${ }^{11}$ Según Gordley, un enfoque teleológico -y, por tanto, aristotélico- del derecho de contratos es la mejor manera de superar las deficiencias de las teorías de contratos basadas tanto en autonomía como en eficiencia, sin abandonar sus intuiciones. ${ }^{12}$ Para Gordley, sin embargo, estas intuiciones están mucho más presentes en el lado de la eficiencia que en el de la autonomía. Así, aunque rechaza algunas de las doctrinas centrales de Law and Economics -como la posibilidad de un 'incumplimiento eficiente' del contrato ${ }^{13}$ - está de acuerdo con que los contratos debieran ser eficientes en el sentido de que los riesgos y cargas de un acuerdo contractual debieran recaer sobre aquella parte que puede soportarlos menos costosamente. Para Gordley, esta regla de asignación de riesgos no solo es eficiente, sino que corresponde exactamente con lo que requiere la virtud aristotélica de la justicia conmutativa. ${ }^{14}$ En contraste, pareciera no haber intuiciones reales derivadas de las teorías de contratos voluntaristas del siglo XIX, la teoría del 'contrato como promesa' de Charles Fried, o incluso del enfoque hegeliano de Peter Benson sobre la autonomía privada y el derecho de contratos. ${ }^{15}$ Ciertamente, Gordley no sostiene que estos autores estén siempre equivocados. Él admite que, a veces, sus conclusiones son compatibles con un enfoque aristotélico, pero, según Gordley, esto ocurre porque estos autores a veces son inconsistentes con sus propias teorías y terminan explicando la autonomía en términos de justicia. ${ }^{16}$

La idea de un precio justo juega un papel central en la explicación del derecho de contratos de Gordley. Según él, si uno asume la deseabilidad de la distribu-

11 Gordley (1991), p. 232: "Podemos tener una teoría de contratos, pero para hacerlo necesitamos los mismos conceptos que los juristas del siglo XIX desecharon. (...) Yo sé que no podríamos resucitar estos conceptos sin grandes cambios al modo en que entendemos el mundo. También soy consciente de que tomaría muchísimo y difícil pensamiento ver cómo estos conceptos debieran ser aplicados. Aunque podemos aprender de los escolásticos tardíos, no podemos simplemente copiarles. (...) A pesar de todo, no veo cómo esa aproximación más amplia sería posible sin utilizar estos conceptos más antiguos."

12 Gordley (2006), pp. 11-12.

13 Gordley (2006), pp. 389-391, 394.

14 Como lo señaló recientemente, sería "económicamente justo". GordLEY y JiANG (2019), p. 1. Véase también Gordley (1991), pp. 318, 323; GoRdLEY y JiANG (2019), passim.

15 Véase Fried (2015); Benson (2001), p. 118.

16 Gordley (1991), p. 242; Gordley (2006), pp. 27-28, 355. 
ción actual de poder adquisitivo entre los miembros de la sociedad, o al menos que ellos no querrían cambiarla por medios distintos a una decisión colectiva, entonces los únicos intercambios jurídicamente exigibles debieran ser aquellos que mantienen la igualdad en el intercambio. La igualdad en el intercambio se conserva cuando las cosas son vendidas y compradas según su precio justo. Para saber el precio justo de una cosa, uno debe preguntar qué precio conservaría la distribución actual de poder adquisitivo. ${ }^{17}$ Según Gordley, este precio es el precio competitivo de mercado -es decir, el precio por el que algo se puede vender en un mercado competitivo-. Bajo condiciones ideales, el precio justo correspondería al precio de equilibrio, esto es, el precio en que las curvas de oferta y demanda se intersecan. Bajo condiciones no ideales, el precio justo sería el precio que más se asemeje al equilibrio del mercado a largo plazo. Si las cargas y riesgos del contrato son asignados eficientemente entre las partes -es decir, si recaen sobre la parte que las puede soportar a menor costo- y estas cargas y riesgos se reflejan proporcionalmente en el precio del contrato, entonces el precio es justo, incluso en caso de que una parte termine perdiendo poder adquisitivo como resultado de la fluctuación de precios del mercado. De este modo, aun cuando en el corto plazo un precio podría parecer injusto, a largo plazo se preserva la justicia, porque los precios de mercado tienden hacia el equilibrio.

Estoy de acuerdo con Gordley en que los precios pueden ser evaluados en términos normativos y que ofrecer una teoría de la justicia en la determinación de precios es relevante para el derecho de contratos. Sin embargo, me parece que su concepción del precio justo no es convincente. ${ }^{18}$ Esto por las siguientes

17 Gordley (2006), p. 404.

18 Aparte de las dos razones principales abordadas en el texto principal, existe también una preocupación algo menor en relación con la conexión entre el concepto del precio justo y el concepto del precio de equilibrio. La preocupación es que ligar el concepto de precio justo al concepto de precio de equilibrio pone una carga innecesaria sobre la teoría del precio justo, puesto que para que una teoría tal funcione, el modelo de equilibrio del mercado también debe funcionar". Pero la última condición puede no ser tan fácilmente satisfecha. En efecto, el modelo de equilibrio del mercado no ha sido inmune a la crítica. Aparte de la famosa crítica de Karl Polanyi de una economía desarraigada, consecuencia del proyecto moderno de producir un mercado que se autorregule (PolanYi (2001), pp. 31, 71 y ss., y passim), y del famoso escepticismo de John Maynard Keynes respecto de los modelos económicos a largo plazo -"este largo plazo es una guía engañosa para los asuntos presentes. A largo plazo todos estamos muertos" (KeYNEs (1971), p. 65)- economistas contemporáneos asociados a una rama de la Escuela Austríaca de economía han argumentado también en contra de la idea de equilibrio sobre la base de que asume racionalidad a largo plazo y estabilidad de elección entre los agentes del mercado, ninguna de las cuales parece probable en mercados reales. Sobre esto, véase en general HaYeK (1945), HaYek (1948), Shackle (1972), Lachmann (1973). Más aun, economistas de todo tipo -iincluso economistas neoclásicos!- han argumentado que el modelo económico de competencia perfecta tiene poco valor como guía normativa. Para 
razones: primero, porque los precios competitivos de mercado no parecen poseer el carácter justificante que Gordley les atribuye -esto es, la capacidad de conservar la actual distribución de poder adquisitivo-. Y segundo, y más importante, porque los precios de mercado corrientes parecen carecer del empuje normativo requerido para proveer un criterio normativo de justicia. Explicaré estos puntos con más detalle en lo que sigue.

El primer problema con la teoría de Gordley, por tanto, tiene que ver con la afirmación de que el precio justo tiene la capacidad de conservar la distribución actual de poder adquisitivo entre los miembros de una sociedad. Sin embargo, pace Gordley, mantener la igualdad en el intercambio no parece ayudar demasiado a conservar la distribución de poder adquisitivo ¿La razón? El poder adquisitivo de cada parte ex ante es diferente de su poder adquisitivo ex post. Consideremos, por ejemplo, la transferencia contractual de bienes o servicios no perdurables o consumibles. Si yo pago por ver un partido de fútbol, o compro una manzana, mi poder adquisitivo después de pagar por el juego o comprar la manzana ha disminuido en comparación con mi poder adquisitivo antes de comprarla. En contraste, el valor del dinero no se pierde, y, por tanto, el poder adquisitivo del vendedor ha aumentado después de la transacción.

Cuando el intercambio involucra bienes no consumibles la situación no es tan distinta, pues incluso cuando los bienes son duraderos, el mero hecho de comprarlos y venderlos disminuye el poder adquisitivo del comprador y aumenta el del vendedor. Si yo compré un libro, ahora solo lo puedo vender como uno 'de segunda mano'. Mi poder adquisitivo, por lo tanto, ha disminuido, mientras que el poder adquisitivo del vendedor ha crecido (en circunstancias normales, el dinero no pierde valor por el intercambio: a nadie le importa tener dinero 'de segunda mano'). La conclusión parece ser que un contrato que mantiene la igualdad de valor en el intercambio podría incluso ayudar a cambiar la distribución en curso al disminuir el poder adquisitivo de una persona y aumentar el de otra: la libertad en los contratos inevitablemente va a cambiar la actual distribución de la riqueza.

Nótese que mi argumento en contra de la explicación de Gordley no es que el precio que mantiene la igualdad de valor entre las cosas intercambiadas -el precio justo- sea incapaz de conservar distribuciones ideales. Mi punto es

críticas de economistas neoclásicos, véase en general ChAmberlin (1956), Clark (1940). Para críticas de economistas post-keynesianos, véase RoBINSON (1969). Lo que estas críticas parecen mostrar -viniendo, por decirlo así, de la 'izquierda', 'centro' y 'derecha'- es que explicar el precio justo en términos de un precio de equilibrio podría conllevar cometer la falacia explicativa de obscurum per obscurius, i.e., explicar un concepto aparentemente oscuro (el precio justo) mediante otro que es más oscuro aun (el precio de equilibrio). 
que el mantener la igualdad de valor en el intercambio es incapaz de conservar cualquier distribución de recursos, ideal o no. Mi argumento se diferencia en este sentido de la crítica de Stephen Smith, según la cual la versión de precio justo de Gordley implicaría que un contrato podría ser justo "solo en sociedades -distintas de cualquiera que conozcamos- que ya son distributivamente justas." 19 Sin embargo, como con razón Gordley mismo ha notado, su argumento no asume una distribución ideal. Gordley admite que no es necesario que un sistema para distribuir recursos funcione perfectamente y que razones pragmáticas podrían justificar una desviación del ideal. Si bien es cierto que Gordley asume que debe haber buenas razones para respetar el patrón de distribución en curso, el hecho de que sea un sistema ideal de distribución de la riqueza no tiene por qué estar entre esas razones. ${ }^{20}$

El punto que yo propongo tiene más en común con el argumento de Wilt-Chamberlain de Robert Nozick, en el que notó "cómo la libertad trastorna los modelos", ${ }^{21}$ esto es, cómo el modo en que cada persona usa su libertad de contratar -uno podría decir su poder adquisitivo- a menudo trastorna los patrones originales de distribución de riqueza. ${ }^{22}$ La respuesta de Gordley a Nozick es que el Estado tiene el deber de intervenir "para prevenir que el ideal se vea comprometido demasiado severamente." ${ }^{23}$ Esto es sin duda cierto, pero creo que aquí Gordley está concediendo lo que no debiera conceder, a saber: que pagar el precio justo y mantener la igualdad en el intercambio no pueden conservar efectivamente la distribución de recursos en marcha. Si la igualdad en el intercambio cumple rol

19 Sмiтh (1996), p. 147; Sмiтh (2004), p. 355.

20 Véase Gordley (2006), p. 13.

21 Nozick (1974), p. 160.

22 NozIcK (1974), p. 161: "Supongamos que Wilt Chamberlain es muy demandado por equipos de baloncesto, siendo una gran atracción de puerta (Además supongamos que los contratos solo duran un año, y que los jugadores son agentes libres.) El firma el siguiente tipo de contrato con un equipo: En cada partido en casa, veinticinco centavos del precio de cada boleto de admisión es para él. (...) La temporada empieza, y la gente alegremente asiste a los juegos de su equipo; compran sus boletos, cada vez dejando unos veinticinco centavos de su precio de admisión en una caja especial con el nombre de Chamberlain. (...) Supongamos que en una temporada un millón de personas asisten a sus partidos en casa, y Wilt Chamberlain termina con $\$ 250,000$, una suma mucho más grande que los ingresos promedio e incluso más grande que los que tiene cualquier otro. ¿Tiene derecho a sus ingresos? ¿acaso es esta nueva distribución (...) injusta? Si es así, ¿por qué? (...) Cada una de estas personas eligió dar veinticinco centavos de su dinero a Chamberlain. (...) Si D1 era una distribución justa, y la gente voluntariamente se movió de esa a D2, transfiriendo partes de sus acciones que les fueron dadas bajo D1 (¿para qué eran si no para hacer algo con ellas?), ¿no es D2 también justa?"

23 Gordley (2001), p. 288. 
alguno en conservar la distribución de poder adquisitivo en curso, este rol parece ser demasiado insignificante para ser usado como una justificación sólida para mantenerla. $^{24}$

Ahora vuelvo sobre mi segunda objeción a la explicación de Gordley. Sugiero que el tomar los precios de mercado corrientes -precios determinados por oferta y demanda- como un criterio normativo para una determinación justa de los precios es problemático. La razón de esto es que los precios de mercado consisten en un agregado de precios en un lapso de tiempo arbitrario que carece de fuerza normativa. Estos precios pueden ser -y quizás en general lo son- $e l$ resultado final de una serie extensa de precios injustos. Para ver esto, consideremos el siguiente ejemplo:

Precios de Vivienda. Compro una casa en Edimburgo bajo circunstancias opresivas y pago el doble del precio de mercado del momento. Esas circunstancias opresivas se replican por todo Edimburgo por seis meses. Al cabo de ese período, el precio de mercado de mi casa es equivalente al precio que pagué por ella. ${ }^{25}$

Precios de Vivienda ilustra una experiencia familiar que también revela la naturaleza problemática de tomar precios de mercado actuales como criterio de justicia. Si el precio que yo pagué por mi casa fue injusto, entonces debe ser verdadero que todos los precios que llevaron al nuevo precio de mercado en Precios de Vivienda fueron también injustos. Pero si el precio de mercado actual da cuenta de la justicia, entonces el nuevo precio de mercado, la suma de una serie de precios injustos debe ser, a pesar de todo, justo. Pero, como observan acertadamente Walsh y Lynch, “¿cómo puede una serie de prácticas injustas relativas a los precios, al ser estas sumadas, llevar a un precio justo?". ${ }^{26}$

24 Para una crítica similar, véase SAPRAI (2010), p. 80. Saprai cree que el problema con la versión de Gordley es que la igualdad en el intercambio causa distribuciones injustas, porque cree -equivocadamente, a mi juicio- que Gordley defiende que "la igualdad en el intercambio está justificada porque promueve distribuciones de recursos justas, o que promueven la autonomía" (p. 80). Por tanto, el hecho de que Gordley conceda que el Estado tiene un deber de intervenir cuando la gente ejercita su autonomía significaría que él concede que la igualdad en el intercambio daña la búsqueda de autonomía. Gordley, sin embargo, no concibe la autonomía de un modo tan absoluto. Véase, por ejemplo, GordLEY (2006), pp. $15-18,25,355,376-379$.

25 Esta es una versión ligeramente modificada de un ejemplo dado por WALSH y LYNCH (2008), p. 135.

26 WaLsh y LYNCH (2008), p. 135 
Otro problema para tomar los precios de mercado corrientes como criterio de justicia radica en la naturaleza indeterminada y maleable de los precios de mercado. Uno podría pensar que las leyes de oferta y demanda son suficientes para determinar qué es lo que cuenta como el precio de mercado de un cierto producto. Pero esto no es así. Consideremos, por ejemplo, el período de tiempo apropiado para determinar el precio de mercado. ¿Qué tan atrás debiéramos ir en el tiempo para calcular el precio de mercado de un determinado bien? ¿Un mes, seis meses, un año, una década? La elección del período de tiempo es una decisión que va a afectar considerablemente a lo que cuente como precio de mercado (y por tanto como precio justo), y sin embargo las leyes de oferta y demanda no dan ninguna guía normativa para elegir entre distintos períodos de tiempo. ${ }^{27}$ Lo mismo aplica para la identificación del mercado relevante para un determinado producto. Los bienes económicos pueden ser descritos de varias maneras distintas (e incluso incompatibles). Una casa puede ser un bien de consumo o un activo financiero. Esto hace que los bienes puedan pertenecer a más de un mercado al mismo tiempo. Es más, pueden incluso pertenecer a diferentes mercados dependiendo del nivel de generalidad de una descripción dada (una botella de Laphroaig puede pertenecer al mercado de 'whiskies', o al mercado de 'whiskies de malta única' o 'single malt', o al de 'whiskies single malt de la región de Islay', o al de 'whiskies single malt de Islay vendidos en Escocia', etc.). La descripción que prefiramos afectará considerablemente cuál será el mercado relevante para un producto, y la especificación del mercado relevante es una decisión que precede a lo que cuenta como oferta y demanda para ese producto. Es una decisión, por tanto, para la que las leyes de oferta y demanda no ofrecen ninguna guía.

Finalmente, hay problemas asociados a la misma idea de demanda. ${ }^{28}$ 'Demanda' es un término general que implica la identidad conceptual entre dos nociones bastante diferentes: deseos ('preferencias') y necesidades. Como nota David Wiggins, los economistas profesionales típicamente conciben las necesidades simplemente como un tipo especial de deseos: una necesidad siempre sería una necesidad de algo que quieres pero que, a pesar de ello, no quieres pagar. ${ }^{29}$ Sin embargo, las necesidades no son un tipo de deseos. La diferencia parece ser que los deseos, como las preferencias, dependen exclusivamente de estados subjetivos, mientras que las necesidades dependen también de un estado objetivo

27 WALSh y LYNCH (2008), p. 135.

28 Para este párrafo, véase Wiggins (1987), pp. 5-9, 25-26; Meikle (1995), pp. 119-121.

29 Wigains (1987), p. 5. 
en el mundo, de lo que sea necesario para que alguien o algo se autorrealice. ${ }^{30}$ A diferencia de la necesidad, el concepto de demanda no tiene esa conexión necesaria con la autorrealización. Wiggins lo explica así:

Si yo quiero tener $x$ y $x=y$, entonces yo no necesariamente quiero tener $y$. Si yo quiero comer una ostra, y esa ostra es la ostra que me condenará al olvido, no se sigue que yo quiero comer la ostra que me condenará al olvido. Pero con las necesidades es distinto. Yo solo puedo necesitar tener $x$ si cualquier cosa idéntica a $x$ es algo que necesito. A diferencia de 'desear' o 'querer', entonces, 'necesitar' no es evidentemente un verbo intencional. Lo que yo necesito no depende del pensamiento o de cómo funcione mi mente (o no solo de estos) sino de cómo es el mundo. Nuevamente, si uno quiere algo porque es $\mathrm{F}$, uno cree o sospecha que es F. Pero si uno necesita algo porque es F, tiene que realmente ser F, crea uno o no que lo es. ${ }^{31}$

A diferencia de los deseos, entonces, las necesidades dependen de un estado objetivo en el mundo. Ese estado objetivo consiste en lo que sea necesario para que alguien o algo se autorrealice. El concepto de demanda no tiene esta conexión necesaria con la autorrealización.

Es más, el concepto de demanda de mercado -como se usa el término en la expresión 'las leyes de la oferta y la demanda'- es aun más problemático y pone aun más presión sobre la idea de tomar los precios de mercado como un estándar de justicia para los precios. Demanda de mercado significa demanda efectiva, es decir, demanda que se registra en el mercado. Es demanda respaldada por dinero. ${ }^{32}$ Esto quiere decir que las necesidades y deseos que no están respaldados por dinero no cuentan como demanda de mercado para un determinado bien. Como acertadamente observan Koehn y Wilbratte, el precio de mercado "excluye a miembros marginalizados de la comunidad cuyos recursos son insuficientes para otorgarles cabida en la curva de la demanda, impidiendo así que sus voces sean tenidas en cuenta respecto a cuál debiera ser el precio predominante [es decir, el precio de mercado]." ${ }^{33}$

30 AnsCOMBE (1958a), p. 7: "Decir que un organismo necesita ese ambiente no es decir, por ejemplo, que tú quieres que tenga ese ambiente, sino que no va a realizarse a menos que lo tenga".

31 Wiggins (1987), p. 6.

32 Véase Meikle (1995), pp. 120-121.

33 Koehn y Wilbratte (2012), p. 506. 


\section{LA IMPORTANCIA NORMATIVA DEL VALOR ECONÓMICO}

La explicación del justo precio de Gordley conlleva una reivindicación del tipo de investigación que representa la tradición escolástica de la doctrina del iustum pretium, esto es, una investigación normativa del valor económico. Sin embargo, las deficiencias de su teoría apuntan a la posibilidad de un replanteamiento y parcial revisión de esa misma tradición. De esto trataré en lo que sigue.

Una alternativa que vale la pena mencionar, aunque no pueda hacerle justicia dentro de los límites de este artículo, es la de desarrollar una explicación de la justicia en la determinación de precios que sea neutral en cuanto al contenido de los mismos. Este es el camino que han tomado autores como Ernest Weinrib y Peter Benson, quienes proponen la justicia correctiva o conmutativa como la estructura normativa formal del derecho privado. ${ }^{34}$ Según Weinrib, la justicia correctiva provee una estructura normativa capaz de unir a demandante y demandado en una única justificación integrada, una que es independiente de cuestiones pertenecientes a la economía, la moral, la política, etc. o a cualquier "noción rica y completa del bien". ${ }^{35}$ Lo mismo aplica para la versión de justicia conmutativa de Benson como la estructura normativa que otorga coherencia al derecho de contratos. ${ }^{36}$ Para ambas posturas, la relevancia normativa del valor económico para el contrato es que permite a las partes contratantes abstraerse de sus necesidades y deseos reales, sirviendo así como un punto de vista común desde el cual pueden verse el uno al otro como iguales. Para Weinrib y Benson, la igualdad en el intercambio tiene que ver con mantener la igualdad entre las partes y sus derechos correlativos para que puedan ser vistos como iguales desde el punto de vista jurídico. ${ }^{37} \mathrm{Al}$ hacer abstracción de las necesidades y deseos reales

34 Más precisamente: Weinrib defiende que la estructura normativa del derecho privado (o, al menos, del derecho de daños) refleja los requerimientos de la justicia correctiva, mientras que Peter Benson defiende que el derecho de contratos refleja los requerimientos de la justicia conmutativa.

35 WeINRIB (1995), p. 80: "El objeto de la ética aristotélica en general es elucidar las excelencias de carácter que marcan el funcionamiento humano apropiado. La justicia correctiva, donde "no hay diferencia si una persona digna ha privado a una persona indigna o viceversa", obviamente se distancia de las preocupaciones generales de Aristóteles. Al ignorar consideraciones de mérito, la justicia correctiva abstrae de las consideraciones que son propias de la noción rica y completa del bien de Aristóteles".

36 Benson (2019), p. 3: "El derecho de contratos (...) puede ser entendido razonablemente como especificando una concepción normativa clara que no solo es extraída de sus principios y doctrinas sino también constituye su idea organizativa, apuntalando y explicando todo el derecho de contratos tanto como sus distintas partes".

37 WeInRIB (2012), p. 130: "La noción de valor calza dentro de la justicia correctiva del siguiente modo. La justicia correctiva trata de las partes que interactúan correlativa- 
de las partes, así como de cualquier otra consideración de hecho, la concepción de valor económico que surge de la explicación de justicia correctiva de Weinrib y de la explicación de justicia conmutativa de Benson tiene la ventaja de desligar el precio justo de preocupaciones sobre justicia distributiva y equilibrio de mercado, como también de las críticas asociadas a la carencia de fuerza normativa de los precios de mercado. Es una explicación de la justicia en la determinación de precios puramente transaccional, no distributiva y basada en la autonomía.

Aquí me gustaría proponer otra alternativa -quizás complementaria- para pensar sobre la justicia en los precios, una que resalta la naturaleza institucional de los precios y ve el precio justo como el precio al que algo puede ser vendido bajo arreglos institucionales justos. En lo que sigue, deseo sugerir que enfocarse

mente como el perpetrador y la víctima de una injusticia. En cuanto gobierna la interacción, la justicia correctiva se aplica a las partes que se afectan la una a la otra al actuar en cosas particulares en el mundo persiguiendo sus necesidades y deseos específicos. Pero en cuanto abarca a ambas partes como situadas correlativamente, la justicia correctiva abstrae de la particularidad de estas cosas y de la especificidad de esas necesidades y deseos a un punto de vista común. El valor es la noción económica que cumple esta función abstractiva.” BENSON (2019), pp. 177-178: "El valor de intercambio siempre presupone dos cosas utilizables cualitativamente diferentes, pero abstrae de estos aspectos cualitativos y trata a los objetos como conmensurables en términos puramente cuantitativos: tanto de $x$ es igual a tanto de $y$. (...) [Un] juicio en términos de precio tiene que ver con valor de intercambio y un juicio relacional como entre los dos lados. (...) El juicio del precio, siendo relacional y quedando bajo la idea de lo razonable [en cuanto opuesto a la idea de lo racional involucrado en el valor de uso], es algo que una tercera parte apropiadamente constituida, como una corte, puede evaluar apropiadamente", p. 181: "Como participantes en la relación de promesa-a-considerar, las partes se ven la una a la otra objetivamente como promitentes y promisarios -como agentes y recipientes- de algo que tiene valor en la contemplación legal. Porque cada una de las partes contribuye desde su lado exactamente del mismo modo que la otra, las partes que interactúan pueden ser vistas en este respecto en términos idénticos como iguales. Aun más, en esta relación y el uno respecto del otro, cuentan como personas mutuamente separadas e independientes, cada una con algo utilizable que está inicialmente bajo su control legítimo exclusivo y que la parte utiliza entregándolo para obtener la consideración prevista a cambio. Este uso de la cosa de uno como medio para obtener la de otro es el poder adquisitivo de la cosa. Ahora, el poder adquisitivo, cuando está generalizado en relación a muchas otras cosas, se transforma en una potencialidad cuantificable que trasciende cualquier transacción y sin embargo al mismo tiempo solo puede ser realizada en y a través de transacciones particulares. Y, suponiendo la existencia de las relaciones de mercado, este poder adquisitivo generalizado es potencialmente medido como el precio de la cosa", p. 387: "El valor es la dimensión de igualdad abstracta de los objetos de una transferencia en paralelo con la igualdad abstracta de las partes que hacen la transferencia", p. 388: "Equivalencia o la igualdad entre cosa y cosa es así el signo transaccional de la igualdad de las personas en cuanto propietarios", p. 389: "Porque este principio estipula la igualdad entre cosa y cosa en las transacciones, expresa explícita y completamente la idea de justicia correctiva o conmutativa; un requerimiento de igualdad aritmética en y para las transacciones". 
en la naturaleza institucional de los precios nos permite acercarnos a la justificación de los precios de una manera más amplia, en la que la justificación de precios incluye más que el cumplir los requerimientos de una única estructura justificante, sea dicha estructura la justicia conmutativa, la autonomía, la eficiencia o la justicia distributiva. Según esta perspectiva, una reformulación adecuada de la teoría del precio justo involucraría rechazar el monismo valórico implícito en los enfoques comunes a preguntas sobre la justicia en los precios y aceptar, en cambio, un pluralismo valórico en la justificación de precios. Este enfoque podría también ayudar a resolver el problema identificado más arriba respecto a la carencia de fuerza normativa de los precios de mercado: la fuerza normativa de los precios de mercado estaría dada por los compromisos normativos incluidos dentro de las reglas que regulan el intercambio. En lo que sigue, argumento a favor de la posibilidad de desarrollar un enfoque institucional y pluralista de la justificación de los precios.

\subsection{Los precios como hechos institucionales}

El punto de partida del enfoque propuesto es que los precios, como los salarios y las tasas de interés, son hechos económicos. Superficialmente, esto podría parecer un punto de partida complejo, puesto que podría parecer que el apuntar a la naturaleza económica de los precios excluye la idea misma de un precio justo. Como argumentó famosamente el filósofo británico R. G. Collingwood, si los precios son hechos fijados por consideraciones económicas (como la oferta y demanda de un determinado producto), entonces la idea misma de un precio justo puede parecer "una contradicción en los términos". ${ }^{38}$ Sin embargo, esto no es necesariamente así. De hecho, hay dos modos de especificar la afirmación de que los hechos económicos son fijados por razones o consideraciones económicas, a saber:

(1) Los hechos económicos son fijados necesariamente por razones económicas.

(2) Los hechos económicos son fijados exclusivamente por razones económicas. ${ }^{39}$

38 Collingwood (1926), p. 174: "Es, por tanto, imposible que los precios sean fijados por cualquier referencia a la idea de justicia o a cualquier otra concepción moral. Un precio justo, un salario justo, una tasa de interés justa, es una contradicción en los términos. La pregunta qué debería obtener una persona a cambio de sus bienes y trabajo es una pregunta absolutamente carente de sentido. Las únicas preguntas válidas son qué puede recibir a cambio de sus bienes o trabajo, y si las debiera vender en absoluto". Citado con aprobación por HAYEK (1990), p. 442.

39 El argumento original de Collingwood en contra de la misma idea de un precio justo es ambiguo respecto de estas dos afirmaciones. Véase, por ejemplo, Collingwood (1926), p. 176: "un salario fijado por cualquier tipo de consideraciones excepto las económicas deja 
Ambas afirmaciones son posiblemente falsas, pero solo (2) es incompatible con la introducción de criterios normativos que permitan a operadores jurídicos distinguir entre precios justos e injustos. De hecho, el derecho no puede asegurar negociaciones justas sin introducir consideraciones morales en la determinación de hechos económicos. Las leyes que protegen la negociación justa regulan el mercado según razones basadas en la justicia, haciendo que de hecho los precios en esos mercados sean determinados, al menos en parte, por consideraciones legales y morales.

Si los precios son sensibles a consideraciones basadas en la justicia introducidas por las reglas e instituciones que regulan las transacciones del mercado $\mathrm{y}$, por tanto, los hechos económicos no carecen completamente de elementos normativos -en otras palabras, si los precios son fijados necesariamente pero no exclusivamente por razones económicas- entonces es posible desarrollar una concepción de precio justo basada en la justicia de esas reglas e instituciones. El precio justo sería, bajo esta concepción, el precio que surge de arreglos institucionales justos respecto al intercambio.

Ciertamente, no podemos seguir este camino si tenemos buenas razones para aceptar la afirmación (2), esto es, si los precios son fijados exclusivamente por consideraciones económicas. Si (2) es cierto, entonces los precios no pueden ser justos ni injustos. Varios economistas solían creer que esta conclusión se seguía lógicamente de la naturaleza libre de valores del discurso económico. ${ }^{40}$ Según esta

de ser un salario" (apoyando la afirmación 1), y p. 174: "Es, por tanto, imposible que los precios sean fijados por cualquier referencia a la idea de justicia o cualquier otra concepción moral" (apoyando la afirmación 2). Esta ambigüedad es lo que le permite defender que un precio justo es una contradicción en los términos (porque los precios son fijados exclusivamente por razones económicas), pero, al mismo tiempo, conceder que una demanda por precios fijados "por la oferta y la demanda en un mercado donde la ley asegure negociaciones justas" (Collingwood (1926), p. 176) es a pesar de todo una demanda racional (porque los precios son fijados necesariamente por razones económicas, pero no exclusivamente: los precios fijados en parte por razones normativas siguen siendo precios mientras sean también fijados por razones económicas). Lo único que Collingwood niega categóricamente -porque se sigue tanto de (1) como de (2)- es la idea de un precio fijado exclusivamente por razones no económicas.

40 Esto es especialmente notorio entre académicos asociados a la Escuela Austríaca de Economía. Véase, por ejemplo, Mises (1966), p. 203: “[La economía] es una ciencia teorética y como tal se abstiene de todo juicio de valor"; HAYEK (1993), p. 231 (defendiendo que el concepto de justicia no es aplicable al orden espontáneo del mercado); HAYEK (1993), pp. 237-238; RobBins (1949), p. vii: “[los juicios de valor] están más allá del alcance de la ciencia positiva"; RobBins (1949), p. 148: "La ciencia económica trata de hechos comprobables; la ética con valoraciones y obligaciones. Los dos campos de investigación no se encuentran en el mismo plano de discurso. Entre las generalizaciones de los estudios positivos y normativos 
perspectiva, el entrelazado de lo descriptivo y lo normativo como el que estaría en el núcleo de la idea de un precio justo distorsionaría nuestra comprensión de los precios. Los precios serían simplemente hechos acerca del mundo, hechos determinados por consideraciones económicas como oferta y demanda, costos de producción, etc. En esta perspectiva, simplemente no hay espacio teórico para la teoría del precio justo: los precios simplemente no son el tipo de cosa de la que se puede predicar la justicia. Del mismo modo que los terremotos, átomos y moléculas, los precios no serían justos ni injustos, porque los hechos -incluidos los hechos económicos- no son ni justos ni injustos.

La imagen de la economía como una disciplina neutral en cuanto a valores, sin embargo, no es acertada. ${ }^{41} \mathrm{El}$ consenso actual entre filósofos de las ciencias sociales es que una teoría puramente descriptiva del actuar humano sin presupuestos de valor es imposible. Amartya Sen ha dedicado el trabajo de toda una vida a defender esta idea, y El Colapso de la Dicotomía Hecho/Valor de Hilary Putnam ha dado mayor soporte analítico a la idea de que cuando se trata del discurso proposicional en la ciencia económica, hechos y valores no pueden ser separados tajantemente. ${ }^{42}$ Como ha afirmado Russel Hardin, no existe algo así como una "elección racional sin valores sustantivos". ${ }^{43} \mathrm{Ni}$ siquiera en la ciencia económica. ${ }^{44}$

hay un abismo lógico fijado que ningún ingenio puede disfrazar y ninguna yuxtaposición de tiempo o espacio puede cruzar".

41 Sobre las conexiones entre lo descriptivo y lo normativo en la ciencia económica, véase Hausman (1992) (2018); Hausman et al. (2016).

42 Putnam (2002).

43 Hardin (2001), p. 57.

44 Estos autores han desarrollado una intuición articulada primero por filósofos del lenguaje ordinario como J. L. Austin en las décadas de 1950 y 1960, y llevada luego un paso más allá por Quine. Los filósofos del lenguaje ordinario habían apuntado a casos híbridos en que los términos que utilizamos en el lenguaje ordinario no son directamente objetivos ni evaluativos (por ejemplo, 'primoroso', 'regordete', o 'cruel'). La defensa de Quine del holismo de creencias -la idea de que nuestras creencias constituyen una red donde cada creencia está atada a todas las demás- le permitió romper la distinción tajante analítico/sintético -y la distinción hecho/valor con ella-. El resultado del enfoque de Quine es que simplemente no hay modo de distinguir de manera exacta entre afirmaciones evaluativas y descriptivas. Esta idea profundiza la significación de los hallazgos de Austin, puesto que términos como 'primoroso', 'regordete' o 'cruel' no serían simplemente casos excepcionales de términos con significado híbrido. Como los términos están unidos holísticamente a componentes tanto evaluativos como objetivos, no debiera ser tan difícil encontrar más casos en que lo descriptivo y lo normativo están constitutivamente entrelazados. Análisis de categorías de raza y género en la ciencia social y biomédica son ejemplos importantes de este tipo de entrelazamiento. La respuesta 
Sea como sea, el punto principal es que la afirmación (2) no se sigue del hecho de afirmar que los precios son hechos económicos. Porque incluso si los precios fueran hechos económicos -hechos fijados por consideraciones económicas- los hechos económicos son hechos del tipo equivocado para las pretensiones de la afirmación (2). Para que (2) sea verdadera, los hechos económicos debieran ser similares a hechos brutos o naturales, dado que estos son el tipo de hechos que no pueden ser determinados por consideraciones morales. Sin embargo, parece extraño pensar en los hechos económicos -y por tanto en los precios- de esta manera. En lo que sigue desarrollo esta afirmación sugiriendo que los precios -cantidades que representan el valor de intercambio de un bien- son mejor concebidos como un tipo de hecho social, a saber, hechos institucionales.

El precio de un bien no es algo que simplemente ocurre sin importar la voluntad de individuo alguno. En este sentido, los precios son diferentes de los hechos puramente naturales como átomos y moléculas: el hecho de que los átomos y moléculas existan no depende de que nosotros tengamos alguna creencia u otra actitud proposicional hacia ellos. Los tipos sociales, por el contrario, están constituidos en parte por las creencias y actitudes proposicionales de aquellos que se involucran en ellos. Este punto puede ser ilustrado con los siguientes ejemplos tomados de MacIntyre: ${ }^{45}$

Lesión Cerebral y Teoría de Partículas. Supongamos que hay una enfermedad generalizada que causa lesiones cerebrales localizadas, resultando en la pérdida de todas nuestras creencias y conceptos acerca de átomos y moléculas, sin dejar ningún rastro de esos conceptos y creencias en nuestro lenguaje y prácticas.

En este caso, no hay duda de que los átomos y moléculas seguirían existiendo después de la pérdida de nuestros conceptos y creencias. Como nota MacIntyre, "nada que ahora sea verdadero en la teoría de partículas sería entonces falso."46 Pero ahora consideremos la siguiente situación:

Lesión Cerebral y Precios. Supongamos que hay una enfermedad generalizada que causa lesiones cerebrales localizadas, resultando en la pérdida de todas nuestras creencias y conceptos acerca de dinero y precios, sin dejar ningún rastro de esos conceptos y creencias en nuestro lenguaje y prácticas.

a los críticos de la teoría del precio justo sería, por tanto, que el análisis económico de los precios debiera también ser añadido a la lista de categorías híbridas como raza y género.

45 MacIntyre (1998), p. 57.

46 Macintyre (1998), p. 57. 
En este caso, el resultado es claro: no existiría nada parecido a precios ni dinero luego de la pérdida de nuestras creencias al respecto. La razón es que los precios no son hechos brutos o naturales. No pertenecen a la misma categoría que los átomos y moléculas. La razón de esto también es clara: la existencia del dinero y de los precios depende de nuestras creencias y actitudes hacia ellos. ${ }^{47}$

Pero hay algo más. Al menos en sociedades complejas y civilizadas como la nuestra, los precios no son simplemente fijados por individuos aislados, ni siquiera por grupos de individuos, según sus propios propósitos o caprichos. ${ }^{48}$ Los precios son producto de instituciones. La compleja red de reglas e instituciones legales que regulan los precios es lo que llamamos el sistema de precios. Sin el sistema de precios, no habría cosa alguna parecida a cantidades que representen valor de intercambio. De hecho, la existencia de precios en una sociedad requiere un medio común de intercambio (dinero), propiedad privada, contratos y otros hechos generados por contextos legales. Los precios, por tanto, como el dinero, ${ }^{49}$ los impuestos y la propiedad privada, ${ }^{50}$ son criaturas del derecho. De hecho, los precios son uno de los casos más paradigmáticos -si no el más paradigmático- ${ }^{51}$ de hechos institucionales, esto es, hechos generados por contextos institucionales.

En la terminología de Searle, los precios son hechos regulados por reglas constitutivas, y no por reglas meramente regulativas. Las reglas regulativas regulan formas preexistentes de comportamiento, mientras que las reglas constitutivas no solo regulan, sino que también "crean o definen nuevas formas de

47 Esto es verdadero respecto al tipo, pero no necesariamente respecto de cada caso individual. Véase SEARLE (1995), p. 32: “[Un] único billete de un dólar podría caer de las imprentas hacia las grietas del suelo y nunca ser usado o considerado dinero en absoluto, pero aún sería dinero. En tal caso, una instancia particular de caso sería dinero, aunque nadie nunca pensara que es dinero o pensó en ella o la usó en absoluto. De modo similar, podría haber un billete falso de un dólar en circulación, aunque nadie supiera que era falso, ni siquiera el falsificador. En tal caso, todo el que usara ese caso particular pensaría que es dinero, aunque no fuera, de hecho, dinero. Acerca de los casos particulares es posible que la gente esté sistemáticamente equivocada". Véase también KHALIDI (2015), p. 98.

48 Hayek creía que este solo hecho hace que la justicia no sea aplicable a los precios de mercado, porque no hay nadie que pueda ser responsable por ellos. Véase HAYEK (1993), pp. 231 y ss. Esto es un non sequitur. Sobre la compatibilidad entre las intuiciones de Hayek sobre precios de mercado y concepciones sustantivas de justicia, véase en general BURCZAK (2006). Véase también Atria (2010).

49 Desan (2016), Rahmatian (2018).

50 Murphy y Nagel (2002).

51 Cuando Elizabeth Anscombe introdujo la distinción entre hechos brutos y hechos institucionales, usó el precio de las papas en un contrato de venta para ilustrar el punto. AnsCOMBe (1958b), p. 69. 
comportamiento." ${ }^{52}$ Tienen la formulación "X cuenta como Y en el contexto Z." ${ }_{53}$ Searle lo explica así:

\begin{abstract}
Donde la regla es puramente regulativa, el comportamiento que se conforma a la regla podría tener la misma descripción o especificación (la misma respuesta a la pregunta “¿Qué hizo él?”) existiera o no la regla, siempre que la descripción o especificación no haga referencia explícita a la regla. Pero donde la regla (o el sistema de reglas) es constitutiva, el comportamiento que se conforma a la regla puede recibir especificaciones o descripciones que no podría recibir si la regla o reglas no existieran..$^{54}$
\end{abstract}

Las reglas de intercambio son reglas constitutivas. No simplemente reconocen y regulan precios preexistentes. Al contrario: dan significado institucional a hechos pre-institucionales respecto del intercambio. Así, es solo cuando tomamos en cuenta el contexto social y legal de trasfondo entregado por las reglas que regulan el intercambio que el hecho bruto de 'A dando dos monedas metálicas a B' se transforma en (esto es, puede ser entendido como) 'A comprando un cuarto de papas a B' o, para usar una terminología levemente distinta, 'A pagando el precio de un cuarto de papas a B. ${ }^{55}$

Si son reglas constitutivas las que definen lo que cuenta como el precio de $x$, entonces la identificación de ciertos hechos, y no de otros, como el precio de $x$ depende de los compromisos normativos entramados institucionalmente que dan forma al sistema de mercado. Las instituciones mismas son formadas por los compromisos normativos de una comunidad política dada. De este modo, un sistema de mercado exclusivamente sesgado hacia rendimientos más altos va a tender a identificar el hecho de que la gente quiera $x$, sea lo que sea, como una razón para poner un precio a $x$, y el hecho de que la gente esté dispuesta a pagar tanto como $£ 100$ por $x$ como una razón para contar $£ 100$ como el precio de $x$. Al contrario, un mercado no interesado exclusivamente en rendimientos más altos contará otros hechos distintos a la voluntad o capacidad del comprador de pagar como hechos relevantes para identificar el precio de una cosa. Por ejemplo, un compromiso normativo por satisfacer necesidades básicas haría que el precio de medicamentos con receta médica no se vea demasiado afectado por cuánto la

52 SeARle (1969), p. 33.

53 Searle (1969), p. 35.

54 SeArle (1969), p. 35.

55 Sobre hechos institucionales, véase especialmente AnsCOMBE (1958b). Véase también Searle (1969), MacCormick \& Weinberger (1986), pp. 49-76; MacCormick (2007), pp. 11-74. 
gente esté dispuesta a pagar por ellos. Por lo tanto, la identificación del precio de un medicamento $x$ como $£ 10$ no respondería al hecho de que algunas personas estén dispuestas a pagar $£ 100$ por $x$.

La demanda por un precio justo es entonces equivalente a la demanda por arreglos institucionales justos en relación con los precios. ${ }^{56} \mathrm{El}$ funcionamiento adecuado del sistema de precios es de gran importancia para la sociedad. Si los precios son demasiado altos o bajos para ciertos bienes, la sociedad entera se ve afectada. El cálculo de precios, por tanto, no es algo que pueda dejarse completamente al arbitrio de un individuo. Uno no puede hacer como le plazca con los precios, porque los precios también 'pertenecen' a la comunidad, de algún modo, y la comunidad tomará ciertas decisiones sobre cuál es el precio correcto a pagar por un determinado producto. En alguna medida, yo compro y vendo como un representante de la comunidad, y no como un individuo aislado. Es por esto que una de las funciones del derecho de contratos es regular las transacciones del mercado según criterios razonables y no según los caprichos de individuos privados. Así, preguntas como bajo qué condiciones, si alguna, accesio, traditio o usucapio cuentan como formas válidas de adquisición de derecho de propiedad, si ciertos bienes pueden ser propiedad privada de alguien en absoluto, qué es lo que implica realmente un derecho de propiedad, cuáles son los requerimientos básicos para que un contrato sea exigible jurídicamente, etc., todas definen de un modo muy específico las condiciones subyacentes bajo las que los bienes debieran ser intercambiados..$^{57}$

La naturaleza institucional de los precios también tiene consecuencias para la afirmación (1), a saber, que los hechos económicos son fijados necesariamente por razones económicas. La consecuencia es que esta afirmación parece ser verdadera solo si estipulamos una definición ad hoc de salarios y precios, una que no sigue la línea del modo en que usamos estos conceptos en el lenguaje ordinario. Ilustremos este punto con un ejemplo:

Precios Fijos. P vive en un país donde los precios son fijados por Sabio Comunista, uno de los ancianos del país que se cree tiene un don divino que le da acceso epistémico al valor de todas las cosas. Sabio Comunista fijó el precio de un cuarto de papas a 22 . P va a comprar un cuarto de papas a un almacén.

56 En una línea similar, LANG (2017), p. 326: "En la medida en que los valores de mercado de activos son una función del orden legal que constituye el mercado (...) la pregunta sobre si el valor de mercado es el 'correcto' (...) se hace indistinguible de la pregunta sobre si el orden legal en que descansa el mercado es normativamente justificable".

57 Cf. Michelon (2014), Michelon (2018). 
El dueño del almacén le dice a $\mathrm{P}$ que el precio para un cuarto de papas es £2. $\mathrm{P}$ paga $£ 2$ por un cuarto de papas.

Alguien que acepte la afirmación (1) -como Collingwood- debe afirmar que Sabio Comunista no fijó el precio del cuarto de papas, que el dueño del almacén no dijo a $\mathrm{P}$ el precio del cuarto de papas, y que $\mathrm{P}$ no pagó el precio por esas papas. Esto parece contraintuitivo. No hay nada que contradiga nuestro uso ordinario de la palabra 'precio' al decir que P pagó un precio por esas papas. Ciertamente, uno podría simplemente aceptar el carácter contraintuitivo de la afirmación e ir en contra de nuestras convenciones lingüísticas acerca del modo en que usamos la palabra 'precio'. Sin embargo, creo que esto sería algo demasiado difícil de aceptar. No veo otra motivación para negar que P pagó el precio de esas papas más que el aferrarse a un punto de definición, de que los precios necesariamente deben ser fijados por la oferta y la demanda. Me parece que esta postura sería como la de alguien que cree que todos los cisnes son blancos, y al serle mostrado un cisne negro, niega que los cisnes negros sean propiamente cisnes.

Nótese que esta objeción colapsa si uno afirma no que un precio fijado exclusivamente por consideraciones no económicas deja de ser un precio, sino que es el precio equivocado a pagar, o que el precio que $\mathrm{P}$ pagó fue injusto. Si aceptamos la afirmación de que el precio justo es el precio que se alcanza bajo condiciones justas de trasfondo del intercambio, entonces el problema con el ejemplo de Precios Fijos es que esos precios serían injustos porque son generados por arreglos institucionales injustos -arreglos que no parecen asegurar negociaciones justas-. Defender que los precios fijos son injustos es compatible con defender que no hay conexión necesaria entre hechos económicos y razones económicas. Una aproximación institucional a los precios nos permite separar afirmaciones sobre hechos económicos de afirmaciones sobre las razones que justifican esos hechos.

\section{OBSERVACIONES FINALES}

Aquí presento cuatro consecuencias de la aproximación a la justificación de los precios que he propuesto, tanto para la versión tradicional de la doctrina del precio justo como para nuestra comprensión general de la justicia contractual:

Primero. Como las instituciones pueden ser conformadas por distintos compromisos normativos, un enfoque institucional nos permite adoptar una aproximación más pluralista a la justificación de precios, que implica una reforma parcial de la aproximación escolástica al iustum pretium y una revisión del compromiso de la teoría del precio justo con la justicia conmutativa como única 
fuente de justificación de los precios. El resultado de este reconocimiento es que, para hacer avanzar la teoría del precio justo, la doctrina escolástica original del precio justo necesita ser modificada parcialmente y complementada por una aproximación más pluralista a la justificación de precios, una que pueda acomodar el hecho de que algunos precios pueden ser justificados normativamente por criterios distintos de la justicia conmutativa. De hecho, una concepción del precio justo que se enfoca en la naturaleza institucional de los precios implica un reconocimiento de que es al menos conceptualmente posible hablar de precios justos $\sin$ invocar igualdad de valor de intercambio o justicia conmutativa como criterio moral: si los precios pre-institucionales no existen, se sigue que tampoco existen los precios pre-institucionalmente justos. Como diría el teólogo escolástico Domingo Báñez (1654), "no existe el precio justo por ley natural, sino solo por ley positiva". ${ }^{58}$

Segundo. Una aproximación institucional a la justicia en los precios podría también ayudar a resolver el problema (identificado más arriba) de la carencia de fuerza normativa de los precios de mercado. En esta perspectiva, la fuerza normativa de los precios de mercado surgiría de su capacidad de manifestar los múltiples compromisos normativos entramados en las reglas que regulan el sistema de precios y el intercambio en el mercado, que pueden coincidir o no con los de la justicia conmutativa.

Tercero. Si queremos hacer que la justificación de los precios en el derecho de contratos sea coherente con una preocupación por el pluralismo valórico, entonces las ineficiencias económicas, la reproducción de patrones no igualitarios de distribución de la riqueza, la opresión sistemática de ciertos grupos dentro de la sociedad y los consiguientes déficits de autonomía de aquellos oprimidos, entre otras inquietudes normativas, deben también ser tomados en cuenta para determinar la justicia de las reglas de derecho de contratos que regulan los precios y el intercambio en el mercado. Esto puede implicar una expansión del rol normativo tradicional asignado al derecho de contratos y a la justicia contractual en relación con los mercados y la justicia distributiva, en que el rol de la justicia contractual es simplemente respetar la autonomía de las partes y de sus correspondientes poderes morales. ${ }^{59}$ Así, además de la justicia conmutativa y del valor de la autonomía, preocupaciones por la eficiencia, la promoción de la justicia

58 Báñez (1654), II-II 771 272: "Nullum est pretium iustum lege naturali, sed solum lege positiva".

59 Para lo que llamo 'postura tradicional', véase BENSON (2019), pp. 395-476. Ciertamente, 'tradicional' quizás no es la mejor palabra para describir la aproximación de Benson a la relación entre derecho de contratos, mercados y justicia distributiva. No me interesa defender la etiqueta. 
distributiva, el igualitarismo, como también de otros compromisos normativos pueden y quizás debieran ser asumidos como parte de las tareas del derecho de los contratos. ${ }^{60}$ Esta afirmación no significa, por supuesto, que no puede haber otras fuerzas normativas en juego, incluso dentro del mismo sistema legal, que podrían ir en la dirección opuesta al desarrollo de un pluralismo valórico en la justificación de precios.

Cuarto. El énfasis en promover un pluralismo valórico en la justificación de precios puede suponer una comprensión del derecho de contratos y de las transacciones privadas distinta tanto del modelo de partes mutuamente desinteresadas negociando para obtener satisfacción de preferencias (Law and Economics) como del modelo de individuos protegidos unos de otros por una esfera de derechos y deberes morales, en que el papel del derecho privado es reflejar una moralidad promisoria ('Contrato como Promesa') ${ }^{61}$ Una aproximación distinta podría ayudar a hacer sentido del surgimiento de varios sistemas jurídicos de reglas que gobiernan transacciones privadas y que desafían la lógica del mercado libre y la libertad de contrato -por ejemplo, el derecho laboral, derecho del consumidor, leyes antitrust, etc.- Estos campos son frecuentemente vistos como intrusiones del ámbito público en el privado. Aunque esta narrativa de intromisiones públicas cada vez más persistentes en la esfera privada no es necesariamente falsa, la omnipresencia creciente de dichas intromisiones podría estar indicando la necesidad de adoptar una narrativa alternativa que capture mejor esta faceta del derecho privado contemporáneo. ${ }^{62}$ Una teoría del derecho de contratos que sea sensible a preocupaciones normativas más allá de la justicia conmutativa podría ayudar a proveer tal narrativa.

Dado que el derecho de contratos opera típicamente bajo el supuesto de igualdad formal o jurídica, un enfoque pluralista a la justificación de precios, en que la justicia conmutativa deja su lugar de honor como la estructura normativa que subyace a los contratos, inevitablemente encontrará resistencia. Sin embargo, si (y en la medida en que) la justificación de precios es reconocida como una parte de la estructura normativa subyacente a nuestras prácticas contractuales -como parte del paisaje normativo del derecho de contratos- dicho reconocimiento puede ayudar a dirigir la atención del derecho privado hacia la omnipresencia de las desigualdades en las transacciones contractuales.

60 Para una afirmación similar respecto al derecho de daños, véase Keren-PAz (2007).

61 La metáfora del reflejo es tomada de SHIFFrin (2012), pp. 250-256.

62 Sobre la necesidad de una nueva narrativa para el derecho privado, véase MicheLoN (2013), pp. 96-100 y passim. Véase también Michelon (2012), reproducido de modo completo en este volumen. 


\section{BIBLIOGRAFÍA CITADA}

Anscombe, G.E.M. (1958a): "Modern Moral Philosophy”, Philosophy, Vol. 33, No. 124, pp. 1-19.

Anscombe, G.E.M. (1958b): “On Brute Facts”, Analysis, Vol. 18, No. 3, pp. 69-72.

Atria, Fernando (2010): “Socialismo Hayekiano”, Estudios Públicos, No. 120, pp. 49-105.

Báñez, Domingo (1654): Decisiones de Iustitia et Iure, tomus quartus.

Benson, Peter (2001): "The Unity of Contract Law", en Benson, Peter (ed.), The Theory of Contract Law: New Essays (Cambridge University Press).

Benson, Peter (2019): Justice in Transactions: A Theory of Contract Law (The Belknap Press of Harvard University Press).

BurCZACK, Theodore (2006): Socialism After Hayek (The University of Michigan Press).

Chamberlin, E.H. (1956): The Theory of Monopolistic Competition: A Re-Orientation of the Theory of Value (Harvard University Press, $7^{\text {a }}$ ed.).

Clark, J.M. (1940): “Toward a Concept of Workable Competition", The American Economic Review, Vol. 30, No. 2, Part 1, pp. 241-256.

Collingwood, R.G. (1926): "Economics as a Philosophical Science", International Journal of Ethics, Vol. 36, No. 2, pp. 162-185.

Dagan, Hanoch y Heller, Michael (2017): The Choice Theory of Contracts (Cambridge University Press).

Desan, Christine (2016): "Money as a Legal Institution" en Fox, David y ERnST, Wolfgang (eds.), Money in the Western Legal Tradition: Middle Ages to Bretton Woods (Oxford University Press), pp. 18-35. 
Deutch, Sinai (1977): Unfair Contracts: The Doctrine of Unconscionability (Lexington Books).

FRIED, Charles (2015): Contract as Promise: A Theory of Contractual Obligation (Oxford University Press, $2^{\text {a }}$ ed.).

Gardner, John (2018): From Personal Life to Private Law (Oxford University Press).

Gordley, James (1981): "Equality in Exchange”, California Law Review, Vol. 69, No. 6, pp. 1587-1656.

GordLey, James (1991): The Philosophical Origins of Modern Contract Doctrine (Clarendon Press).

GoRDLEY, James (2001): “Contract Law in the Aristotelian Tradition” en BENSON, Peter (ed.), The Theory of Contract Law: New Essays (Cambridge University Press), pp. 265-334.

Gordley, James (2006): Foundations of Private Law: Property, Tort, Contract, Unjust Enrichment (Oxford University Press).

Gordley, James (2015): “The Just Price: The Aristotelian Tradition and John Rawls", European Review of Contract Law, Vol. 11, Issue 3, pp. 197-219.

Gordley, James y JiAng, Hiao (2019): "Fairness and the Law of Contract", SSRN Scholarly Paper ID 3324001, en: <https://papers.ssrn.com/abstract $=3324001>($ visitada el 19 de junio de 2019).

HARDIN, Russell (2001): “The Normative Core of Rational Choice” en MäKI, Uskali (ed.), The Economic World View: Studies in the Ontology of Economics (Cambridge University Press), pp. 57-74.

Hausman, Daniel (1992): The Inexact and Separate Science of Economics (Cambridge University Press).

Hausman, Daniel; McPherson, Michael y Satz, Debra (2016): Economic Analysis, Moral Philosophy, and Public Policy (Cambridge University Press).

Hausman, Daniel (2018): "The Bond Between Positive and Normative Economics", Revue d'Economie Politique, Vol. 128, Issue 2, pp. 191-208. 
HAYEK, Friedrich A. (1945): “The Use of Knowledge in Society”, The American Economic Review, Vol. 35, No. 4, pp. 519-530.

HAYEK, Friedrich A. (1948): “The Meaning of Competition”, en HAYEK, Friedrich A., Individualism and Economic Order (University of Chicago Press), pp. 92-106.

HAYEK, Friedrich A. (1990): The Constitution of Liberty (Routledge).

HAYEK, Friedrich A. (1993): Law, Legislation and Liberty: A New Statement of the Liberal Principles of Justice and Political Economy (Routledge).

Kahneman, Daniel; KNETSCh, J.L. y ThaleR, R. (1986): "Fairness as a Constraint on Profit Seeking: Entitlements in the Market", The American Economic Review, Vol. 76, No. 4, pp. 728-741.

KaPlow, Louis y Shavell, Steven (2006): Fairness versus Welfare (Harvard University Press).

Keren-Paz, Tsachi (2007): Torts, Egalitarianism and Distributive Justice (Ashgate).

Keynes, J.M. (1971): The Collected Writings of John Maynard Keynes: Volume 4. A Tract On Monetary Reform (Second edition, Cambridge University Press) (Macmillan for the Royal Economic Society).

KhaLIDI, Muhammad Ali (2015): “Three Kinds of Social Kinds", Philosophy and Phenomenological Research, Vol. 90, Issue 1, pp. 96-112.

Koenn, Daryl y Wilbratte, Barry (2012): “A Defense of a Thomistic Concept of the Just Price”, Business Ethics Quarterly, Vol. 22, Issue 3, pp. 501-526.

KornhauSER, Lewis (2017): "The Economic Analysis of Law", The Stanford Encyclopedia of Philosophy, Fall 2017, en $<$ https://plato.stanford.edu/archives/fall2017/entries/legal-econanalysis/> (vistado el 19 de junio de 2019).

Kronman, Anthony y Posner, Richard (1979): The Economics of Contract Law (Little, Brown and Company).

Lachmann, L.M. (1973): Macro-Economic Thinking and the Market Economy: An Essay on the Neglect of the Micro-Foundations and Its Consequences (Institute of Economic Affairs). 
LANG, Andrew (2017): "Market Anti-Naturalisms" en DesAutels-STEIn, Justin y Tombins, Christopher (eds.), Searching for Contemporary Legal Thought (Cambridge University Press), pp. 213-329.

MacCormick, Neil (2007): Institutions of Law: An Essay in Legal Theory (Oxford University Press).

MAcCormick, Neil y WeInBerger, Ota (1986): An Institutional Theory of Law: New Approaches to Legal Positivism (Reidel).

MacInTyre, Alasdair (1998): "Social Science Methodology as the Ideology of Bureaucratic Authority" en KNight, Kelvin (ed.), The MacIntyre Reader (Polity Press), pp. 53-72.

MeIKLE, Scott (1995): Aristotle's Economic Thought (Oxford University Press).

Michelon, Claudio (2012): “The Public Nature of Private Law?" en MicheLon, Claudio; Clunie, Gregor; McCorkindale, Christopher y Psarras, Harris (eds.), The Public in Law: Representations of the Political in Legal Discourse (Ashgate).

Michelon, Claudio (2013): "The Public, the Private, and the Law" en MAC Amhlaigh, Cormac; Michelon, Claudio and Walker, Neil (eds.), After Public Law (Oxford University Press), pp. 83-102.

Michelon, Claudio (2014): "Virtuous Circularity: Positive Law and Particular Justice”, Ratio Juris, Vol. 27, Issue 2, pp. 271-287.

Michelon Claudio (2018): "What Has Private Law Ever Done for Justice?" Edinburgh Law Review, Vol. 22, Issue 3, pp. 329-346.

Mises, Ludwig (1966): Human Action: A Treatise on Economics (Liberty Fund, $3^{\mathrm{a}}$ ed.).

Murphy, Liam y Nagel, Thomas (2002): The Myth of Ownership: Taxes and Justice (Oxford University Press).

Nozick, Robert (1974): Anarchy, State, and Utopia (Basic Books).

Polanyi, Karl (2001): The Great Transformation: The Political and Economic Origins of Our Time (Beacon Press, $2^{\mathrm{a}}$ ed.). 
Posner, Richard (2014): Economic Analysis of Law (Wolters Kluwer Law \& Business, $9^{\mathrm{a}}$ ed.).

Putnam, Hilary (2002): The Collapse of the Fact/Value Distinction and Other Essays (Harvard University Press).

RAHMATIAN, Andreas (2018): "Money as a Legally Enforceable Debt", European Business Law Review, Vol. 29, Issue 2, pp. 205-236.

RipsteIn, Arthur (2016): Private Wrongs (Harvard University Press).

Robisns, Lionel (1949): An Essay on the Nature and Significance of Economic Science (Macmillan \& Co Ltd, $2^{\mathrm{a}}$ ed.).

Robinson, Joan (1969): The Economics of Imperfect Competition (MacMillan, $2^{\mathrm{a}}$ ed.).

SAPRAI, Prince (2010): “Against Equality of Exchange”, King's Law Journal, Vol. 21, Issue 1, pp. 71-95.

SeArle, John R. (1969): Speech Acts: An Essay in the Philosophy of Language (Cambridge University Press).

SEARLE, John R. (1995): The Construction of Social Reality (Allen Lane Penguin Press).

SHACKLE, George L.S. (1972): Epistemics \& Economics: A Critique of Economic Doctrines (Cambridge University Press).

ShIFfrin, Seana Valentine (2012): “Are Contracts Promises?" en Marmor, Andrei (ed.), The Routledge Companion to Philosophy of Law (Taylor and Francis), pp. 241-258.

Smith, Stephen (1996): "In Defence of Substantive Fairness", Law Quarterly Review, Vol. 112, pp. 138-158.

Smith, Stephen (2004): Contract Theory (Oxford University Press).

Study Group on a European Civil Code (Study Group) y Research Group on EC Private Law (Acquis Group) (2009): Principles, Definitions and Model Rules of European Private Law. Draft Common Frame of Reference (DCFR) (Sellier. European Law Publishers, ed. Outline). 
VoyIAKIs, Emilios (2017): Private Law and the Value of Choice (Hart Publishing).

WALSH, Adrian y LyNCH, Thomas (2008): The Morality of Money: An Exploration in Analytic Philosophy (Palgrave Macmillan).

WeInRIB, Ernest (1995): The Idea of Private Law (Oxford University Press, ed. rev.).

WeInRIB, Ernest (2012): Corrective Justice (Oxford University Press).

Wiggins, David (1987): Needs, Values, Truth: Essays in the Philosophy of Value (Oxford University Press, $3^{\mathrm{a}}$ ed.).

\section{LEGISLACIÓN CITADA}

\section{United States of America:}

Restatement (Second) of Contracts (1981). 